\title{
The initialization of the learner model combining the Bayesian networks and the stereotypes methods
}

\author{
Mouenis Anouar Tadlaoui ${ }^{1 *}$, Rommel Novaes Carvalho ${ }^{2}$ and Mohamed Khaldi ${ }^{1}$ \\ Abdelmalek Essaâdi University, Faculty of Sciences, Lirosa, Tétouan, Morocco ${ }^{1}$ \\ University of Brasília, Department of Computer Science, Brasília, Brazil ${ }^{2}$
}

Received: 25-August-2017; Revised: 02-November-2017; Accepted: 03-November-2017

(C)2017 ACCENTS

\begin{abstract}
Learner modalization in adaptive systems contains several indicators, such as domain knowledge, learning performance, goals, tasks, background, learning styles and learning environment. Even if there are several methods for initializing the learner model, such as the stereotype model, or learner profiles, these models does not manage the side of uncertainty in the dynamic modeling of the learner. The main hypothesis of this article is the initialization of the learner model based on the combination of the Bayesian networks and the stereotypes methods. To achieve this objective, it is necessary to ask why and how to initialize a model of the learner by combining the method of stereotypes with Bayesian networks? What steps can be taken to move from the learner information gathering phase to the initialization of a learner model in a comprehensive way? We focus in this article on the first two steps in the process of adaptation, collecting data about the user, and initiating the learner model. In order to carry out a complete initialization of this model, a combination of the stereotypes method to process the content of the specific domain of information, and the Bayesian networks to process the contents of the independent domain of information have been used.
\end{abstract}

\section{Keywords}

Learner model, Bayesian networks, Stereotypes, Adaptive hypermédia educational systems, Cognitive diagnosis.

\section{Introduction}

The user module is an essential component for Adaptive e-Learning systems. The term adaptation in e-learning systems involves the selection and the manner of presentation of each learning activity as a function focused on the entity having of knowledge and skills and other information given by each subject taught [1].

Despite the different attempts to model the learning that is characterized by a dynamic aspect, we always find it difficult to achieve this goal [2]. The proposed approaches give us just a static view of the learner model, and don't manage the two domains of this model equally [3]. It doesn't cover the three stages of development of the learner model: the data collection, the initialization of the model and its update [4].

In practice this model is in full development (the learner knowledge is evolving in the same module) [5].

\footnotetext{
*Author for correspondence
}

Thus, a dynamic view is essential. Therefore, in order to monitor the behavior of the learner in real time during a learning situation; we must adopt a model for dynamic management of the learning model, including the two types of domains of this model, and it three stages of initialization [6].

The structure of this paper consists of combining Bayesian networks with stereotypes method to initialize the learner model in adaptive hypermedia educational systems (AHES). To achieve that goal, we will firstly return to the notions and definitions of the learner's model, the process of its development, its domains of information and their contents, and then we will concentrate on the Bayesian networks, their definitions and their rules of construction. Then, we will explain our approach for the initialization of the learner model based on a combination of the stereotype method and the Bayesian networks, precisely the field of intervention of each method and their expected results. Finally, and in order to disclose the validity of our hypothesis; we will present the experiments and the tests carried out. The results obtained from these experiments presented in this work are all arguments in favor of our 
hypothesis. This combination also promotes its reuse in the step of updating the learner model in different adaptive educational hypermedia systems.

\section{Materials and methods}

2.1The initialization of the learner model in AHES We will dedicate this part of the initialization of the model of the learner in the AHES, beginning by describing the process of initialization of the model that we will propose by combining the Bayesian networks and the stereotypes in order to achieve a complete initialization in all aspects.

2.1.1The process of initializing the learner model The initialization of the learner model represents the process of gathering information about the learner and transferring this information to the model. This process of initialization represents a major problem for adaptive systems. In this section, we will present our process of collecting data about the learner. The learner model can be initialized in three ways, using explicit questions, initial tests, or the method of stereotypes [7].

Figure 1 shows our proposed process for collecting learner data. The use of two methods simultaneously to gather all the information related to the learner, which will give us a more complete learner model, and this will be reflected on the adaptation of the system to the needs of the learner in a more precise way have been proposed.

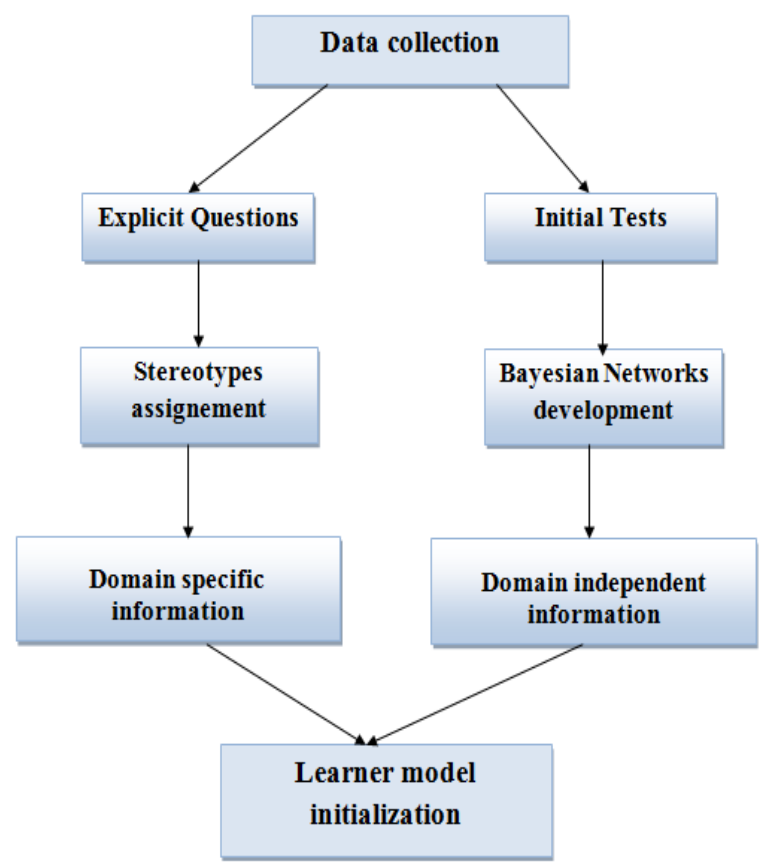

Figure 1 The process of initializing the learner model
To achieve this goal, we will use two methods for data collection: explicit questions, and initial tests. In order to gather specific information about the learner that makes up the learner's profile, such as personal information, goals, cognitive skills and preferences, we will base this process on explicit questions. It may guide us in assigning each learner to a well-defined stereotype.

For independent learner information, which reflects its level of knowledge and skills for each module in the system, and which represent the key element for system adaptation? We base this process on initial tests, using Bayesian networks as a formalism to properly represent and manage this information in a probabilistic way.

2.1.2Building the learner profile

Initial learner models are often constructed directly by direct questioning of the learner as soon as he or she enters the system and before he or she has been affected into a learning situation. This method is considered very effective for collecting general information about the learner, the problem encountered using this method, is to find a number of appropriate questions to collect consistent information about the learner taking into consideration that the increase of the number of questions can irritate the learner and increase the declination to the system, which can create a situation where the learner leaves the system and never returns. The dependence on this method alone does not allow sufficient extraction of information for the initialization of a learner model [8].

In order to answer the problem of numbers of questions for the extraction of the data on the learner, we propose to use the stereotypes method to group the learners in categories. Stereotypes would concede a very powerful method to provide considerable information based on initial observations.

\section{a) Stereotypes: Definition and characteristic}

In general, stereotypes represent a category or a group of learners. There are two types of stereotypes: Fixed and default.

In fixed stereotypes, the learner is assigned a predefined stereotype at an abstract level. For example: in a module, learners are divided into four groups, corresponding to four levels (novice, beginner, advanced and expert) each level is more difficult than the previous one: After obtaining individual information such as: knowledge, experience and skills, the system attributes to each 
learner one of the four levels and it will never change in this type of stereotype.

In default stereotypes, the system is more flexible. Therefore, first, the learner is assigned to the initial stereotype. This means that the initial stereotype has a "default" value. The system will observe the learners and collect their performance data, test results during the learning process. Finally, the system will modify the initial stereotype to new stereotype more appropriate. Simply, the default stereotype setting is gradually replaced by a more accurate stereotype for the learner.

There are three important elements in a stereotype: triggering, inference and retraction:
- Trigger is used to activate a stereotype. In other words, it is a condition for assigning a stereotype to the learner.

- Inference is an engine, responsible for inferring information related to the learner

- Retractions are the conditions used to disable the stereotype of the learner. There is a circumstance

\section{b) The process of building a learner profile}

Figure 2 shows an overall view of the process of building our profile of each learner. As soon as the learner enters the system, the system will ask the learner a set of questions about his personal information, and then he will receive answers to these questions. These responses is then be stored in the system.

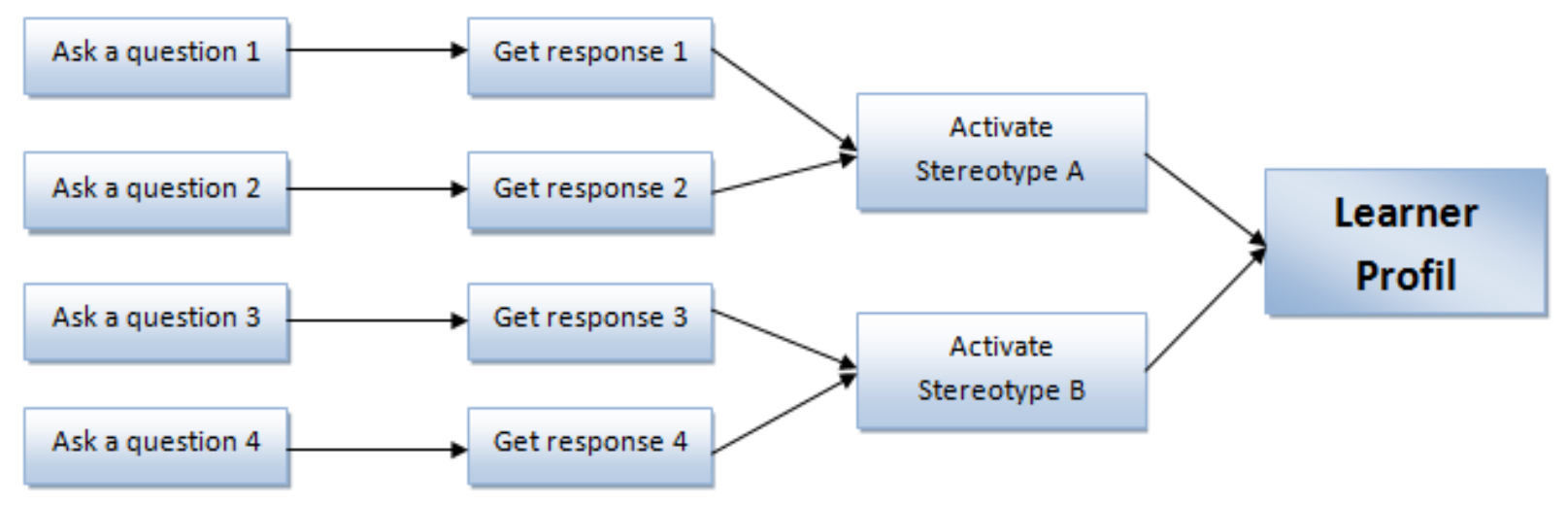

Figure 2 The process of building a learner profile

After one or more of these responses (which represent triggers or stereotypes), one or more stereotypes will be activated. These stereotypes will gather a group of learners into specific categories according to well-defined characteristics. All of these stereotypes will build a profile of the learner.

2.1.3The development of stereotypes

The use of stereotypes in learner modeling began with GRUNDY [9], which allows the learner to give him a personal description. This description is used by the system to trigger a predefined trigger in the system. Depending on the trigger triggered, the system adapts to the needs of the learner.

Assuming an S-stereotype that aims to collect learner information to initialize part of a learner model in an adaptive system, and learner responses to explicit system questions as components $\{c j\}$ of the system, each of these components represent aspects of the learner during his or her time in the system.

\section{a) Activation of stereotypes}

The activation of each stereotype is related to a set of triggers $\{\mathrm{tSi}\}$, where each trigger $\{\mathrm{tSi}\}$ is a Boolean expression based on a component $\{\mathrm{cj}\}$ or a function of a set of components $\mathrm{f}(\{\mathrm{ck}\})$ model of the learner.

The first action of a stereotype is related to the validity of the learner's response to an explicit question posed by the system. Thus:

if $\exists i, t S_{i}=$ true $\rightarrow$ active $(S)$

Meaning that when a trigger $\{\mathrm{tSi}\}$ has become true, stereotype $\mathrm{S}$ will be activated.

\section{b) The retraction of stereotypes}

There is also a series of retractions $\{\mathrm{rSi}\}$ in the stereotype method that is considered the basis on which an S stereotype is deactivated.

A stereotype $\mathrm{S}$ is deactivated if a retraction condition $\{\mathrm{rSi}\}$ has become true:

$\exists j, r S_{i}=$ true $\rightarrow$ desactive $(S)$ 
2.2Initial testing with Bayesian networks

For a complete collection of learner information, we will use the initial test method, which will allow us to initialize the learner model in a complete way taking into account all aspects and components of this model. By asking the learner to take the tests, the initial parameters of the learner model can be obtained through the analysis of the results of these tests.

The problem encountered in using this method is the control of the length of the tests proposed to the learner. Length or redundancy in these tests may cause the learner to leave the system. To solve this problem the Bayesian networks as a method to construct these initial tests have been used. On the one hand, we can benefit from the power of this method for managing the probabilistic aspect of the learner's information which will guide us towards the construction of a well-structured set of tests and, on the other hand, to realize a comprehensive analysis of the domain information of the learner model based on the results of each test and on the real-time monitoring of the learner during each proposed test.

- The specification of the structure of the model: in which we define the nodes and the orientation of the arcs for all the components of our network.

- The specification of the values of variables, in which we will calculate the value tables of the variables of each node of our network.

2.2.1The structure specification of the model

To illustrate the stages of development of Bayesian networks to model the initial tests for the collection of data on the learner, we will begin by defining the nodes of our network. Our network consists of an initial "Initial Test" node, which is composed of several parent nodes representing the modules taught in the Specialized Master "Pedagogical Engineering Multimedia" (Ingénierie Pédagogique Multimédia) at Abdelmalek Essaâdi University; in our work, we chose three modules with different criteria to test the validity of our hypothesis (Project Management, Digital Image Processing, Human Machine Interaction). Each of these nodes is also composed of the parent nodes, the links of these nodes are preliminary relations:

- Initial tests: This node allows the collection of all the data from the tests of each module the learner chosen to take.

- Project management: the learner, and before following the training of this module, it is led to take an initial test to measure its level of knowledge and competence, the latter consists of two types of evaluation:

* Knowledge: the learner must answer to more than 10 questions to measure how much of his knowledge. This part of the assessment reflects the evaluation of the learner's knowledge.

* Ability: this is a written proof that responds if the learner can apply the knowledge acquired in the module. This type of evaluation reflects the part of the learner's know-how.

- Digital Image Processing: the learner, and before following the training of this module, he is led to take an initial test to measure his level of knowledge and skill, the latter consists of two types of evaluation:

* Knowledge: the learner must answer to more than 10 questions to measure how much of his knowledge. This part of the assessment reflects the evaluation of the learner's knowledge.

* Ability: this is a written proof that responds if the learner can apply the knowledge acquired in the module. This type of evaluation reflects the part of the learner's know-how.

- Human machine interaction: the learner, and before following the training of this module, he is led to take an initial test to measure his level of knowledge and skill, the latter consists of two types of evaluation:

* Knowledge: the learner must answer to more than 10 questions to measure how much of his knowledge. This part of the assessment reflects the evaluation of the learner's knowledge.

* Ability: this is a written proof that responds if the learner can apply the knowledge acquired in the module. This type of evaluation reflects the part of the learner's know-how.

The value that measures the relative importance of each condition varies from 0 to 1 , the values of each evaluation element are defined by the teacher.

In other words, it is the weight of the arc from the parent node to the child node. All weights regarding the child variable will build its conditional probability table (CPT). The sum of the weights of all arcs at / from each child / parent node in case of hidden variables / proofs should be 1. This means that each weight is standardized. The relationship between the target variable $(\mathrm{T})$ and the variable of the proof (E) must be from $\mathrm{T}$ to $\mathrm{E}$ because the process that calculates the posterior probability of the target variable is evidence of diagnostic knowledge. So, if 
variable of evidence has no child and its parents must be target variables. There are two types of relationships:

- Prerequisite relationships between the target variables.

- Diagnostic relationships of the target variables to the proof. The mastery of concepts (targets) effects on the confidence of evidence. However, if the learner has failed an exam, he is not sure of his lack of knowledge or ability because he can make an unexpected mistake.

2.2.2The specification of the values of the variables Once the network structure is presented, it is easy to define the values of the variables. Figure 3 shows the Bayesian network of initial tests for the initialization of the learner model in an educational hypermedia system.

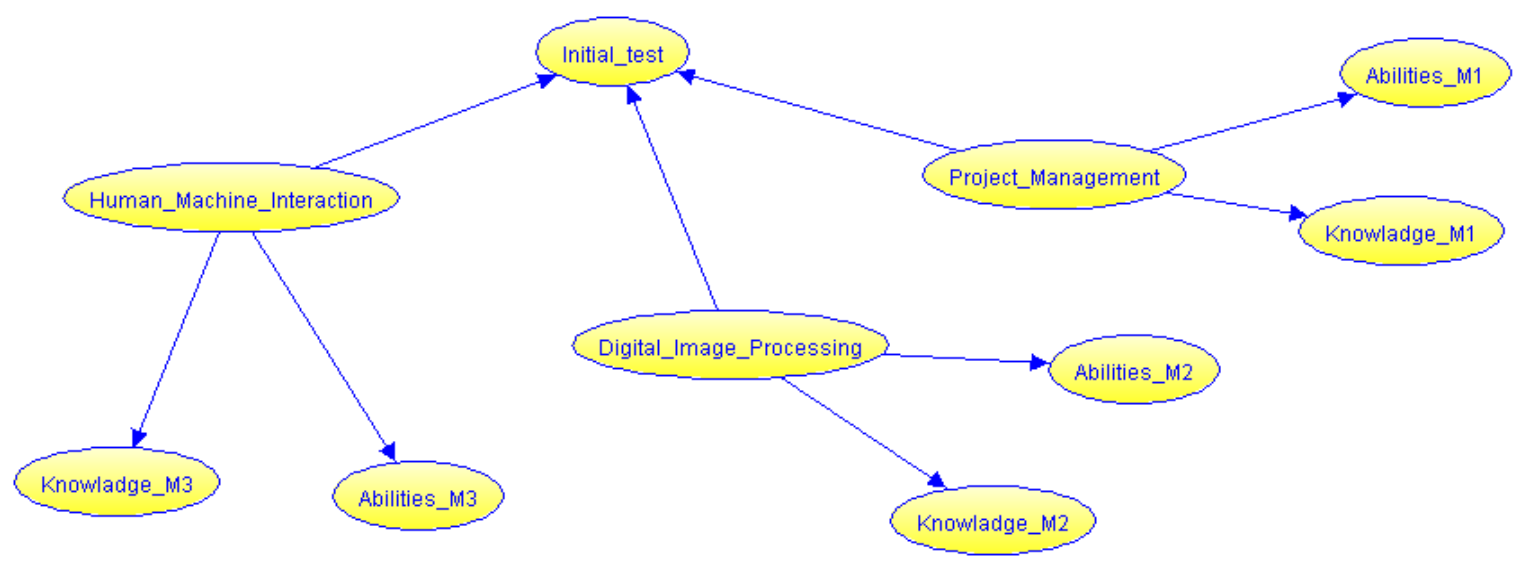

Figure 3 The Bayesian network of initial tests

In the developed Bayesian network, we observe that the initial test of node (I) consists of three parents: digital image processing $(\mathrm{T})$, project management $(\mathrm{G})$ and human machine interaction $(\mathrm{M})$ corresponding to three reports of preliminary weights: $\mathrm{w} 1=0.1$, w $2=$ 0.5 , w $3=0.4$. The conditional probability of (I) is calculated as follows:

$P(I \mid T, G, M)=w 1 * h 1+w 2 * h 2+w 3 * h 3$

Thus:

$$
\begin{aligned}
h 1 & =\left\{\frac{1 \text { if } T=I}{0 \text { otherwise }}\right. \\
h 2 & =\left\{\frac{1 \text { if } G=I}{0 \text { otherwise }}\right. \\
h 3 & =\left\{\frac{1 \text { if } M=I}{0 \text { otherwise }}\right.
\end{aligned}
$$

We should indicate that $\{\mathrm{I}, \mathrm{T}, \mathrm{G}, \mathrm{M}\}$ is a complete set of mutually exclusive variables, whose variable is also random and binary.

By generalizing on the formula below, it is that:

$$
P(X=1 \mid Y 1, Y 2 \ldots Y n)=\sum_{i=1}^{n} w i * h i
$$

Where $h 1=\left\{\frac{1 \text { if } Y i=X}{0 \text { otherwise }} \quad\right.$ With random binary variables $X, Y$ i. Obviously, $\mathrm{P}(\operatorname{not} \mathrm{X} \mid \mathrm{Y} 1, \mathrm{Y} 2, \ldots, \mathrm{Yn})=$ $1-\mathrm{P}(\mathrm{X} \mid \mathrm{Y} 1, \mathrm{Y} 2, \ldots, \mathrm{Yn})$, a) The conditional probability table of the node "Initial test"

Table 1 shows the CPT of each child node of the initial test of the parent node:

Table 1 Conditional probability table of the "Initial Test" Node

\begin{tabular}{lllll}
\hline $\mathbf{T}$ & $\mathbf{G}$ & $\mathbf{M}$ & $\mathbf{P}(\mathbf{J}=\mathbf{1})$ & $\begin{array}{l}\mathbf{P}(\mathbf{J}=\mathbf{0}) \\
\mathbf{1 - P}(\mathbf{J}=\mathbf{1})\end{array}$ \\
\hline 1 & 1 & 1 & $1.0\left(0.1 * 1+0.5^{*} 1+0.4 * 1\right)$ & 0.0 \\
1 & 1 & 0 & $0.6\left(0.1 * 1+0.5^{*} 1+0.4 * 0\right)$ & 0.4 \\
1 & 0 & 1 & $0.5(0.1 * 1+0.5 * 0+0.4 * 0)$ & 0.5 \\
1 & 0 & 0 & $0.1(0.1 * 1+0.5 * 0+0.4 * 0)$ & 0.9 \\
0 & 1 & 1 & $0.9(0.1 * 0+0.5 * 1+0.4 * 1)$ & 0.1 \\
0 & 1 & 0 & $0.5(0.1 * 0+0.5 * 1+0.4 * 0)$ & 0.5 \\
0 & 0 & 1 & $0.4(0.1 * 0+0.5 * 0+0.4 * 1)$ & 0.4 \\
0 & 0 & 0 & $0.0(0.1 * 0+0.5 * 0+0.4 * 0)$ & 1.0 \\
\hline
\end{tabular}

Because the concepts T, G, M does not have prior knowledge of comprehension, their CPTs are specified as prior probabilities obeying a uniform distribution as shown in Table 2 (mean value assigned 0.5 in most cases)

Table 2 Parent conditional probability table of the "Initial Test" node

\begin{tabular}{cccccc}
\hline $\mathbf{P}(\mathbf{T}=\mathbf{1})$ & $\mathbf{P}(\mathbf{T}=\mathbf{0})$ & $\mathbf{P}(\mathbf{G}=\mathbf{1})$ & $\mathbf{P}(\mathbf{G}=\mathbf{1})$ & $\mathbf{P}(\mathbf{M}=\mathbf{1})$ & $\mathbf{P}(\mathbf{M}=\mathbf{1})$ \\
\hline 0.5 & 0.5 & 0.5 & 0.5 & 0.5 & 0.5 \\
\hline
\end{tabular}


b) The conditional probability table of the node "Digital image processing"
Table 3 represents the CPT of each child node of the parent node digital image processing:

Table 3 Conditional probability table of the "Digital image processing" node

\begin{tabular}{|c|c|c|}
\hline$K$ & $\mathbf{P}(\mathrm{J}=1)$ & $\begin{array}{c}\mathbf{P}(\mathbf{J}=\mathbf{0}) \\
1-\mathbf{P}(\mathbf{J}=1)\end{array}$ \\
\hline 1 & $0.8(0.8 * 1)$ & 0.2 \\
\hline 0 & $0.0(0.8 * 0)$ & 1.0 \\
\hline $\mathbf{A}$ & $\mathbf{P}(\mathrm{J}=\mathbf{1})$ & $\begin{array}{c}\mathbf{P}(\mathbf{J}=0) \\
1-\mathbf{P}(\mathbf{J}=1)\end{array}$ \\
\hline 1 & $0.2(0.2 * 1)$ & 0.8 \\
\hline 0 & $0.0(0.8 * 0)$ & 1.0 \\
\hline
\end{tabular}

c) The conditional probability table of the "Project Management" node

Table 4 shows the CPT of each child node of the parent node Project management:

Table 4 The conditional probability table of the "Project Management" node

\begin{tabular}{ccc}
\hline $\mathbf{K}$ & $\mathbf{P}(\mathbf{J}=\mathbf{1})$ & $\begin{array}{r}\mathbf{P}(\mathbf{J}=\mathbf{0}) \\
\mathbf{1 - P}(\mathbf{J}=\mathbf{1})\end{array}$ \\
\hline 1 & $0.6(0.6 * 1)$ & 0.4 \\
0 & $0.0(0.6 * 0)$ & 1.0 \\
\hline $\mathbf{A}$ & & \\
\hline 1 & $\mathbf{P}(\mathbf{J}=\mathbf{1})$ & $\mathbf{P}(\mathbf{J}=\mathbf{0})$ \\
0 & & $\mathbf{1 - P}(\mathbf{J}=\mathbf{1})$ \\
\hline & $0.4(0.4 * 1)$ & 0.6 \\
& $0.0(0.6 * 0)$ & 1.0 \\
\hline
\end{tabular}

\section{d) The conditional probability table of the node} "Man Machine Interaction"

Table 5 shows the CPT of each child node of the parent node. Man Machine Interaction:

Table 5 The conditional probability table of the node "Man Machine Interaction"

\begin{tabular}{ccc}
\hline $\mathbf{K}$ & $\mathbf{P}(\mathbf{J}=\mathbf{1})$ & $\begin{array}{r}\mathbf{P}(\mathbf{J}=\mathbf{0}) \\
\mathbf{1 - P}(\mathbf{J}=\mathbf{1})\end{array}$ \\
\hline 1 & $0.7(0.7 * 1)$ & 0.3 \\
0 & $0.0(0.7 * 0)$ & 1.0 \\
\hline & & \\
\hline $\mathbf{A}$ & $\mathbf{P}(\mathbf{J}=\mathbf{1})$ & $\mathbf{P}(\mathbf{J}=\mathbf{0})$ \\
& & $\mathbf{1 - P}(\mathbf{J}=\mathbf{1})$ \\
\hline 1 & $0.3(0.3 * 1)$ & 0.7 \\
0 & $0.0(0.7 * 0)$ & 1.0 \\
\hline
\end{tabular}

\section{Results}

After developing our Bayesian network combining the superposition model, we will try to validate and justify our approach by testing it in a real context. To do this, we chose the first year class of IPM (Master's
Degree in Engineering Pedagogy Multimedia). Before presenting our experimental results, using the developed Bayesian network, we have presented the framework in which our approach has been evaluated and the parameters on which the evaluation is based. Next, the combined Bayesian network and its performance evaluation have been performed.

\subsection{Evaluation metrics}

The results of our tests and the performance of the learner module modeled using Bayesian networks have been presented in this section. The UnBBayes software [10] allows us to evaluate dynamically and in real time the performance of each node in our network. Here are the parameters we used to evaluate our Bayesian network:

Here are the metrics used to evaluate our Bayesian network:

- The global confusion matrix (GCM) calculated for the selected target node and all selected proof nodes.

- Probability of correct classification (PCC): the probability of a correct classification is calculated from the global confusion matrix taking into account all the control nodes of the Bayesian network.

- Marginal CPC (MPCC): the probability of a correct classification, it is calculated from the global confusion matrix, taking into account all the control nodes in the Bayesian network other than that presented in the row.

- Marginal improvement (MI): the probability of a correct classification is calculated from the global confusion matrix, taking into account all the nodes of evidence in the Bayesian network and obtained by adding the node presented in the row the remains of other nodes. 
- Individual PCC (IPCC): the probability of a correct classification calculated from the LCM by considering only the evidence presented in the row.

- Cost rate: the individual probability of a correct classification on the cost ratio.

\subsection{The combined Bayesian network}

Before presenting the evaluation results of each node of our Bayesian network representing the initial tests to initialize the learner model in an adaptive system, we will begin by presenting the combined Bayesian network through the UnBBayes software.

Figure $4 a$ gives us a diagram of the combined network, with the marginal variables of each node of our network developing; we can observe the change in the marginal variables of each node of our network, simply by changing one or the more marginal variable of one or more parent nodes of the selected node.

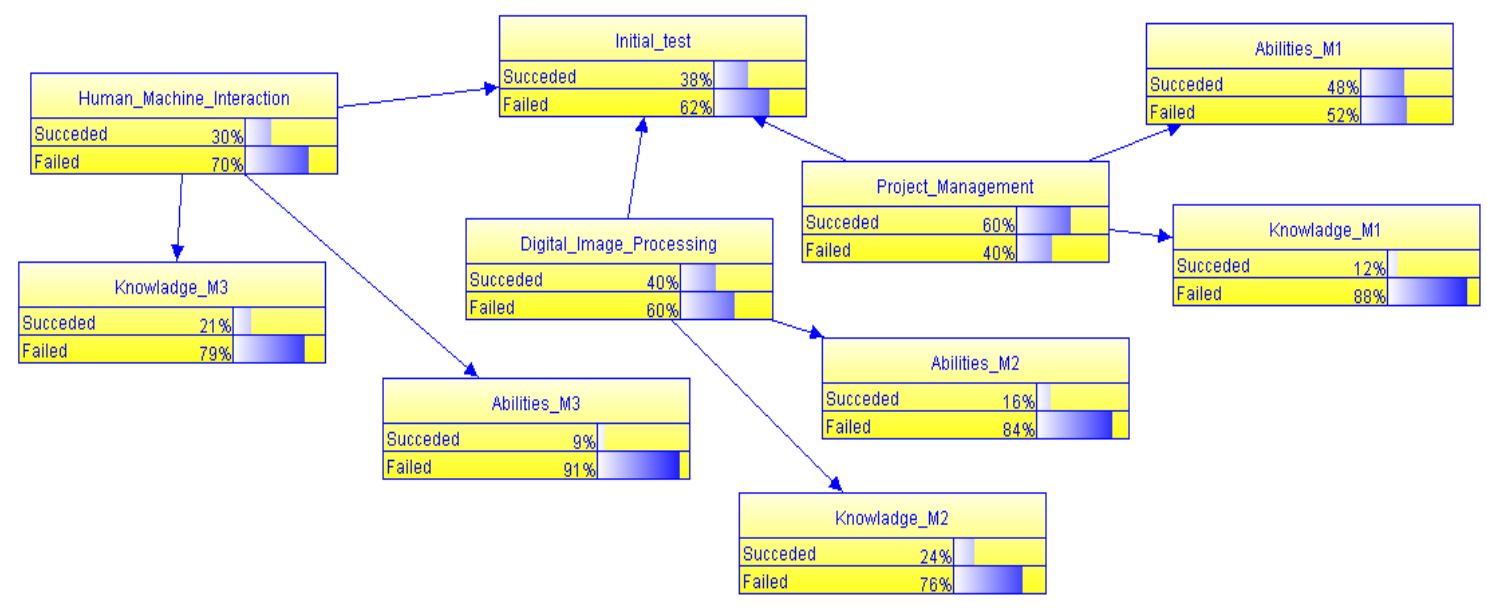

Figure 4a The Bayesian network compiled of the learning situation

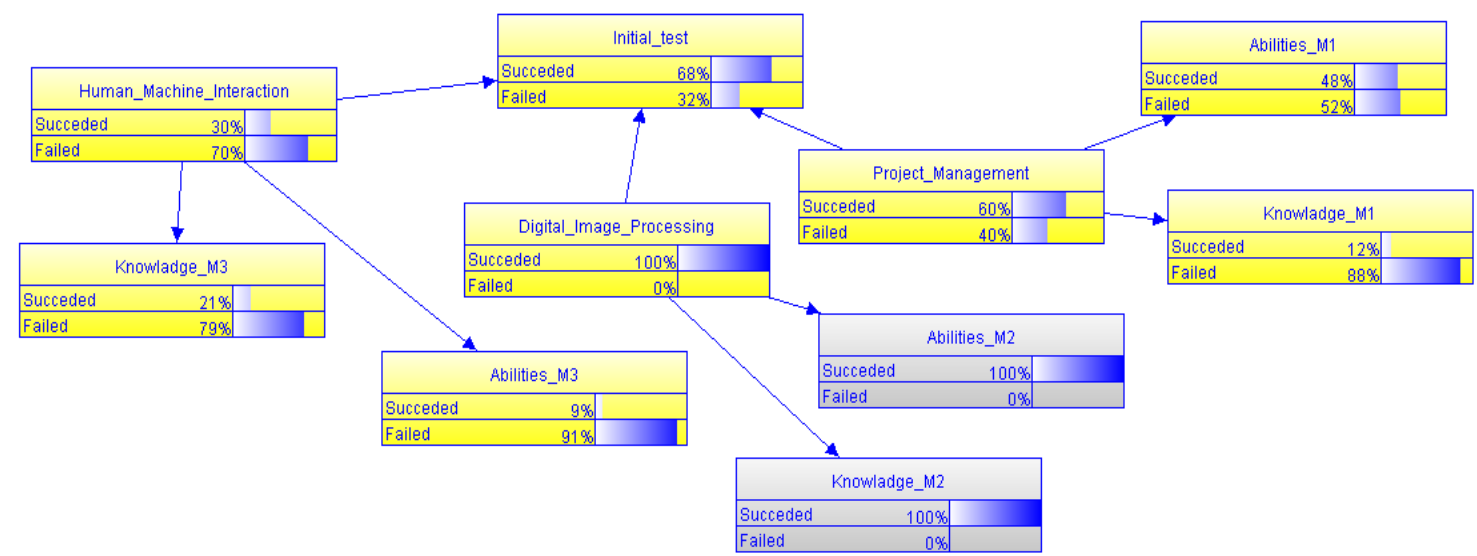

Figure 4b The Bayesian network compiled of the learning situation

In Figure $4 b$, the Bayesian network obtained after the change of the marginal variables in the two parent nodes "Knowledge" and "Skills" of the node "Project management" has been presented.

The marginal variables of these two nodes are changed from the initial percentage to $100 \%$, which means that the learner to pass both evaluations successfully. We note that the marginal variables of 206 the nodes "Project management" and "Initial test" will also change. For the "Project management" node, the marginal success variable is high up to $100 \%$, and for the "Initial test" node, the marginal success variable is raised to $68 \%$. All this means that the success of each learner in the initial set of tests is primarily related to his / her success in the tests of each module. 


\subsection{Evaluation of node performance}

In order to evaluate the performance of our developed Bayesian networks, the performance of each node in our network, using the UnBBayes software and the evaluation metrics presented in the previous section has been evaluated.

3.3.1Evaluation of the node "Digital image processing"
For the "Digital image processing" node, there are two parent nodes: "Knowledge" and "Abilities", choosing the "Digital image processing" node as a target node and the parents as nodes of results shown in Figure 5.

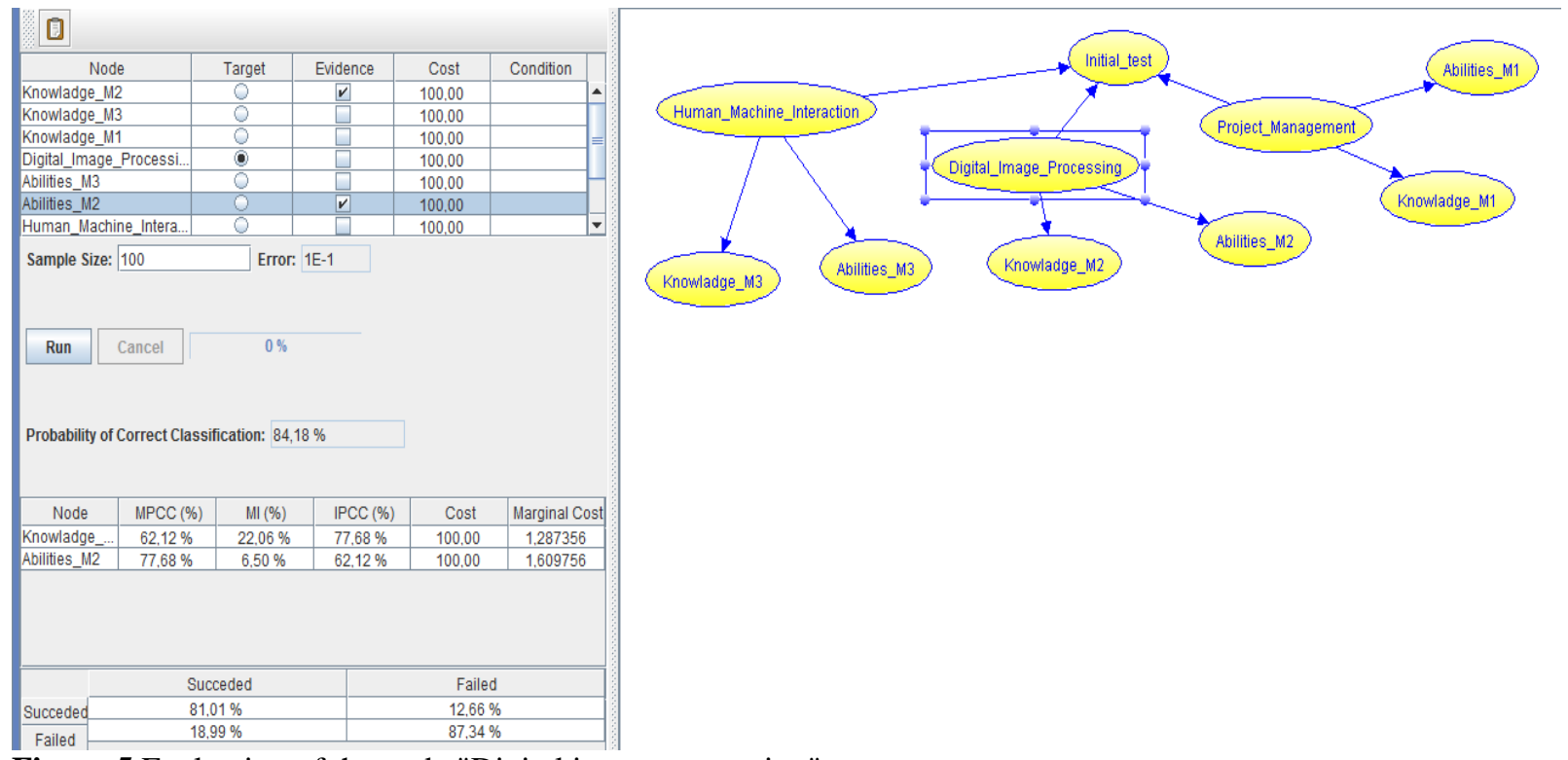

Figure 5 Evaluation of the node "Digital image processing"

According to the results of the results table, we find that by adding nodes of evidence in our evaluation of the target node, the probability percentage of correct classifications increase. By measuring the probability of a correct classification of each node, we see how each node contributes independently to the classification. In this evaluation, the node "Abilities" is the node that contributes the most has been found.

We find how each node contributes according to the set of nodes in front of them. In this evaluation, the marginal improvement of the node "Abilities" means that the influence of this node is greater with respect to the target node. We also note that, although the cost is the same, the marginal cost of the two different sensors, the sensor, which in most cases represents the marginal cost of the variables of the "Abilities" node.

All this reflects that the success of a learner in this module of digital image processing is based more on his success in the test that relates to these abilities, more than the test that relates to his knowledge. The system can therefore, on the basis of its results to define the level of knowledge of the learner (based on the result of the knowledge test), his level of skill (based on the result of the ability test), and also to deduce the learning style and preferences of the learner in this learning situation. In order to adapt according to the profile of each learner, the affect the appropriate module has been shifted.

3.3.2Evaluation of the node "Project management"

For the "Project Management" node, there are two parent nodes: "Knowledge" and "Abilities", choosing the "Project Management" node as a target node and the parents as nodes of evidence, in Figure 6.

According to the results of the results table, we find that: by adding nodes of evidence in our evaluation of the target node, the probability percentage of correct classifications increase. By measuring the probability of a correct classification of each node, we see how each node contributes independently to the classification. In this evaluation, we find that the node "Knowledge" is the node that contributes the most. 


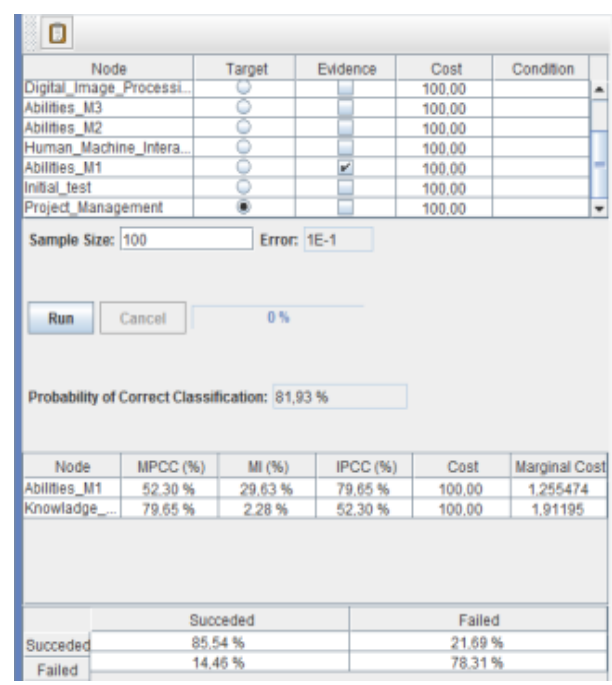

Figure 6 Evaluation of the node "Project management"

We find how each node contributes according to the set of nodes in front of them. In this evaluation, the marginal improvement of the "Knowledge" node means that the influence of this node is greater than the target node. We also note that, although the cost is the same, the marginal cost of the two different sensors, the sensor, which in most cases represents the marginal cost of the variables of the Knowledge node.

All this reflects on the success of a learner in this module of project management is based more on his success in the test that concerns this knowledge, more what the test relates to his abilities. The system can therefore, on the basis of its results to define the level of knowledge of the learner (based on the result of the knowledge test), his level of skill (based on the result of the ability test), and also to deduce the learning style and preferences of the learner in this learning situation, in order to adapt according to the profile of each learner, we shift affect the appropriate module.

3.3.3Evaluation of the node "Interaction man machine" In the human machine interaction node, there are two parent nodes: "Knowledge" and "Abilities", selecting the «human machine interaction» node as a target node and the parents as nodes of evidences, in Figure 7.

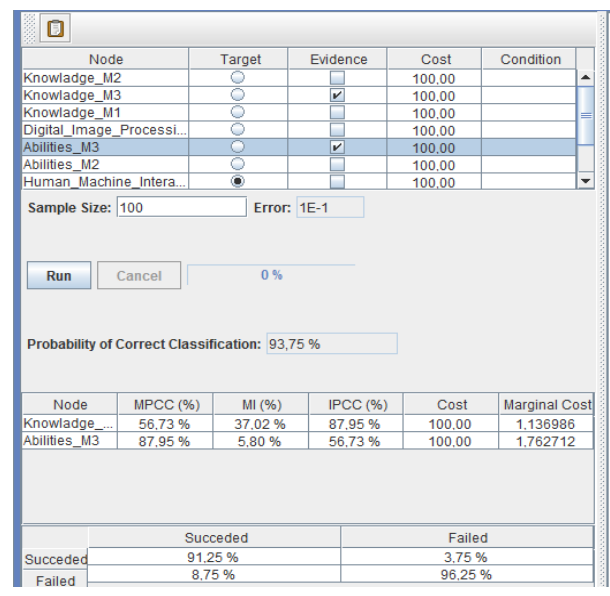

Figure 7 Evaluation of the node "Interaction man machine"

According to the results of the results table, we find that by adding nodes of evidence in our evaluation of the target node, the probability percentage of correct classifications increases. And by measuring the probability of a correct classification of each node, we see how each node contributes independently to 
the classification. In this evaluation, we find that the node "Abilities" is the node that contributes the most. We find how each node contributes according to the set of nodes in front of them. In this evaluation, the marginal improvement of the "Abilities" node means that the influence of this node is greater than the target node. We also note that, although the cost is the same, the marginal cost of the two different sensors, the sensor, which in most cases represents the marginal cost of the variables of the Abilities node.

All this is reflected in the success of a learner in this module human machine interaction is based more on his success in the test that concerns this knowledge, more than the test that relates to his abilities. The system can therefore, on the basis of its results to define the level of knowledge of the learner (based on the result of the knowledge test), his level of skill (based on the result of the ability test), and also to deduce the learning style and preferences of the learner in this learning situation, in order to adapt according to the profile of each learner, we shift affect the appropriate module.

\subsection{Evaluation of the full Bayesian network}

After the validation of each node of the Bayesian network of our model of the learner. This section presents the validation results of the complete Bayesian network.

Figure 8 presents the validation results of the Bayesian network in a complete way. In this evaluation of the network, we will only ask the learner to successfully pass the two initial tests of the two modules "human machine interaction" and "project management". The marginal variations of the initial node "Initial test" will in this case have a value of $61.47 \%$. Changing one of these two nodes will affect the marginal variables of our network in a probabilistic way.

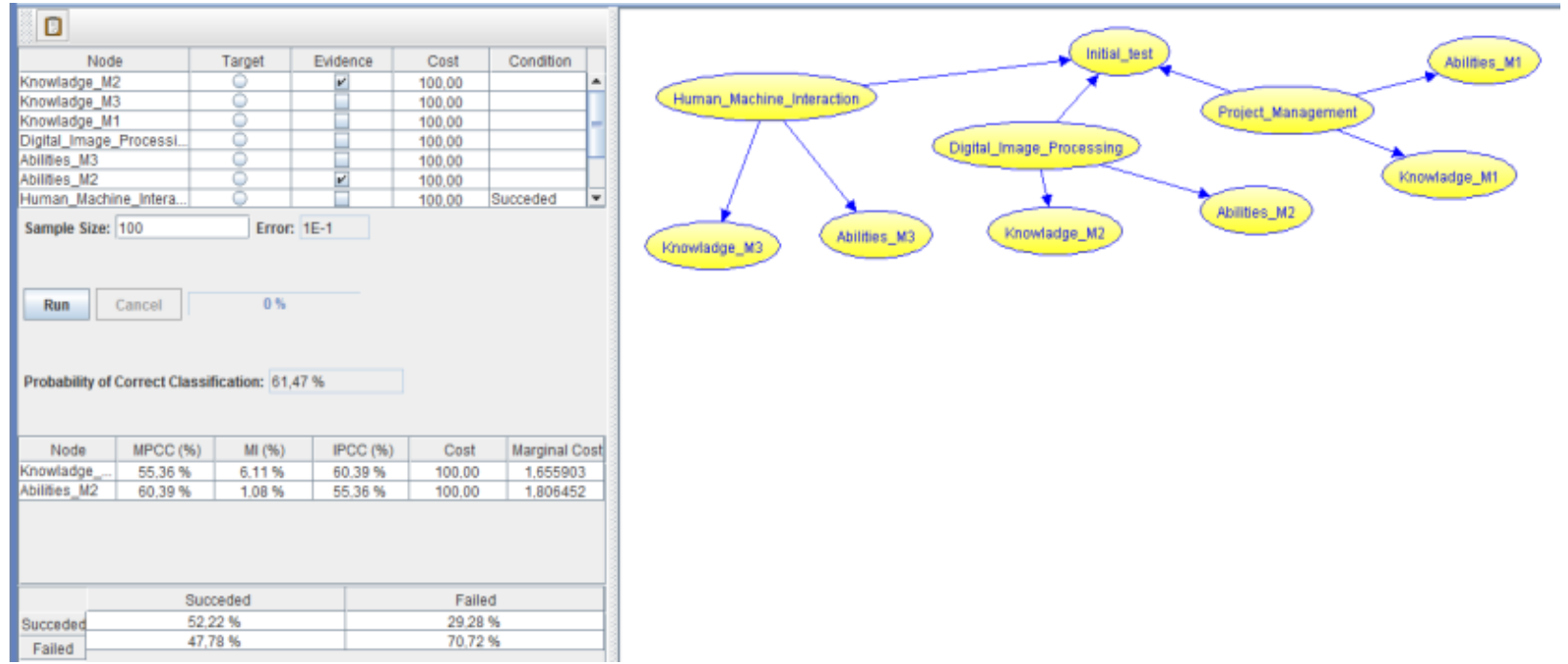

Figure 8 The evaluation results of the "initial test" node of the developed Bayesian network

Based on the results and the validation of each node of the Bayesian network, we were able to manage the network in a global way.

When the learner enters the system and before assigning the module to his / her needs and according to his / her level of knowledge and skills, the learner will be required to take a set of initial tests concerning all the modules available in adaptive educational hypermedia system. Each of these modules consists of two types of evaluation; an assessment based on the learner's knowledge, and an assessment based on these abilities.

The results from these two types of tests will enable us to define the level of knowledge, the level of competences, the learning styles and other information about each learner in our system; for example, if a learner passes the initial test of the "Digital image processing", we will demonstrate his level of knowledge of the learner in this module based on the results of the "Knowledge" type tests and his skill level in this module based on the results 
of the "Skills" tests, and we can therefore deduce from the results of both types of tests the appropriate learning style of this learner in this module.

\section{Discussion}

The approach presented in this paper combines the method of stereotypes for the collection of contents of the specific information domain, based on the explicit questions posed to the learner after entering the system, using the Bayesian network method for the collection of contents of the independent information domain, based on the initial tests the learner is led to take before assignment in a specific learning situation.

Table 6 shows different contents of the learner model with their description and explanation, grouping each component according to the appropriate information model, and the methods used for the initialization of the set of these contents.

Table 6 Summary of methods used for the initialization of the learner model

\begin{tabular}{|c|c|c|c|c|c|}
\hline \multirow[t]{2}{*}{ Model } & \multirow[t]{2}{*}{ Cotenant } & \multirow[t]{2}{*}{ Description } & \multicolumn{3}{|c|}{ Modeling technique used } \\
\hline & & & Stereotypes & $\begin{array}{l}\text { Bayesian } \\
\text { networks }\end{array}$ & $\begin{array}{l}\text { Other } \\
\text { methods }\end{array}$ \\
\hline \multirow{8}{*}{$\begin{array}{l}\text { Domain specific } \\
\text { information }\end{array}$} & Personal information & Name; Email; Password & $\mathrm{X}$ & - & - \\
\hline & Demographic data & Age; Sex; Nationality & $X$ & - & - \\
\hline & $\begin{array}{l}\text { Academic } \\
\text { background }\end{array}$ & Previous studies and courses taken & $X$ & - & - \\
\hline & Qualifications & Previous certificates taken & $X$ & - & - \\
\hline & Trace of personality & Psychological profile of the learner & $\mathrm{X}$ & - & - \\
\hline & Deficiencies & Learner vision, using glasses or not. & $X$ & - & - \\
\hline & Learning styles & Determination of learning styles & - & $\mathrm{X}$ & $\mathrm{X}$ \\
\hline & Cognitive capacities & $\begin{array}{l}\text { Determination of the learner cognitive } \\
\text { skills }\end{array}$ & $\mathrm{X}$ & - & - \\
\hline \multirow{7}{*}{$\begin{array}{l}\text { Domain } \\
\text { independent of } \\
\text { information }\end{array}$} & $\begin{array}{l}\text { Knowledge } \\
\text { background }\end{array}$ & $\begin{array}{l}\text { Learner previous knowledge on a } \\
\text { learning situation }\end{array}$ & - & $\mathrm{X}$ & - \\
\hline & Objectives & The objectives of the learning situation & - & $\mathrm{X}$ & - \\
\hline & $\begin{array}{l}\text { Navigation } \\
\text { description }\end{array}$ & $\begin{array}{l}\text { Kipping a register of each page } \\
\text { completed }\end{array}$ & - & $\mathrm{X}$ & - \\
\hline & Knowledge acquired & $\begin{array}{l}\text { A collection of knowledge transformed } \\
\text { on concepts for every learning } \\
\text { situation. }\end{array}$ & - & $\mathrm{X}$ & - \\
\hline & Evaluation results & $\begin{array}{l}\text { Keeping a register for every test data or } \\
\text { assessment result. }\end{array}$ & - & $\mathrm{X}$ & - \\
\hline & $\begin{array}{l}\text { Learner Aptitude and } \\
\text { interest }\end{array}$ & $\begin{array}{l}\text { Definition of the capacity and aptitude } \\
\text { to use the system }\end{array}$ & - & $\mathrm{X}$ & $X$ \\
\hline & Deadline extend & $\begin{array}{l}\text { Determination of the duration of every } \\
\text { learning situation. }\end{array}$ & - & $\mathrm{X}$ & - \\
\hline
\end{tabular}

The results of tests and validation presented in the previous sections show that this combination of the collection of data on the learner in an adaptive educational hypermedia system will allow us to initiate the learner model in a clear way and complete.

As we have shown in the table above, even if some content of the learner model such as the learning styles, and learner skills, can be represented using Bayesian networks, we will propose to a combination of Bayesian networks with other modeling methods of the learner model, and precisely the superposition model that addresses the problem of the dynamic management of the learner model by representing the learner's knowledge as a subset of knowledge of the system.

The work that we presented in this paper, is a major step towards a full modalization of the learner in adaptive educational hypermedia, a modalization that's include the two domains of information of this model in a probabilistic way.

\section{Conclusion and future work}

Our approach suggests that the combination of the stereotype method and the Bayesian networks is an appropriate approach for the initialization of the learner model in the adaptive educational hypermedia systems. The experiments presented in this article are 
all arguments in favor of our hypothesis on the probabilistic initialization of the learner model, using all the nodes as sensors to measure and evaluate the modulus of the learner.

This approach may encounter difficulties in the case of a large number of data stored in the system for which we have proposed in previous works the use of multi Bayesian networks to try to cover all the data even if the number of the data stored is pupil [11].

We see mainly two directions in which to continue this work. On the one hand, the method will be integrated in the step of updating the learner model in an adaptive educational hypermedia system. On the other hand transform the Bayesian networks developed for the management of the learner model in a machine-readable language, such as ontologies, or using probabilistic ontologies as a formalism that gives us the possibility of combining Bayesian networks with ontologies [12].

\section{Acknowledgment}

None.

\section{Conflicts of interest}

The authors have no conflicts of interest to declare.

\section{References}

[1] Yudelson M, Brusilovsky P, Zadorozhny V. A user modeling server for contemporary adaptive hypermedia: an evaluation of the push approach to evidence propagation. International conference on user modeling 2007(pp. 27-36). Lecture Notes in Computer Science. Springer, Berlin, Heidelberg.

[2] Tadlaoui MA, Aammou S, Khaldi M, Carvalho RN. Learner modeling in adaptive educational systems: a comparative study. International Journal of Modern Education and Computer Science. 2016; 8(3):1-10.

[3] Brusilovsky P, Sosnovsky S, Shcherbinina O. User modeling in a distributed e-learning architecture. International conference on user modeling 2005 (pp. 387-91). Lecture Notes in Computer Science. Springer, Berlin, Heidelberg

[4] Zaitseva L, Boule C. Learning systems in professional training. Workshop "industry meets research" within the conference interactive computer aided learning ICL 2005 Villach, Austria.

[5] Mouenis AT, Souhaib A, Mohamed K. Learner modeling based on Bayesian networks. In e-learninginstructional design, organizational strategy and management. In Tech; 2015.

[6] Han B. Student modelling and adaptivity in web-based learning systems. Massey University, New Zealand. 2001.

[7] Self JA. Formal approaches to student modelling. In student modelling: the key to individualized knowledge-based instruction 1994 (pp. 295-352). Springer, Berlin, Heidelberg.

[8] Tsiriga V, Virvou M. Initializing student models in web-based ITSs: a generic approach. In proceedings of the international conference on advanced learning technologies 2003 (pp. 42-6). IEEE.

[9] Rich E. Building and exploiting user models. In proceedings of the international joint conference on artificial intelligence 1979 (pp. 720-2). Morgan Kaufmann Publishers Inc.

[10] Costa PC, Ladeira M, Carvalho RN, Laskey KB, Santos LL, Matsumoto S. A first-order Bayesian tool for probabilistic ontologies. In proceedings of the twenty-first international FLAIRS conference 2008 (pp. 631-6). Association for the Advancement of Artificial Intelligence.

[11] Tadlaoui MA, Khaldi M, Aammou S. Towards a learning model based on Bayesian networks. In international conference on education and new learning technologies 2014 (pp. 3185-93). IATED.

[12] Mouenis AT, Mohamed K, Souhaib A. Towards probabilistic ontology based on Bayesian networks. International Journal of Software and Web Sciences. 2014; 10(1): 102-6.

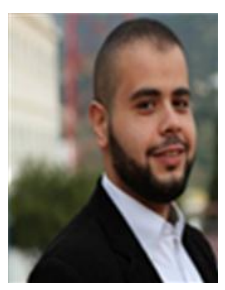

Mouenis Anouar Tadlaoui is a $\mathrm{PhD}$ candidate in Computer Science, at the Laboratory of Informatics, Research Operational and Statistic Applied (LIROSA) at Faculty of Sciences, Abdelmalek Essaadi University. He has a Master degree in Instructional Design Multimedia engineering at The École Normale Supérieure of Martil, Morocco in 2013. His current research focuses on: E-learning, Adaptive Hypermedia Systems, Artificial Intelligence, and Bayesian Networks.

E-mail: tadlaouimouenis@gmail.com

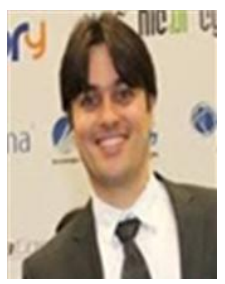

Rommel Novaes Carvalho finished his Postdoctoral at George Mason University (GMU) in the area of artificial intelligence, data mining, uncertainty, and knowledge discovery in May 2012. During the 3 years of his $\mathrm{PhD}$, he was a Graduate Research Assistant in the Department of Systems Engineering and Operations Research at GMU, Virginia, USA. He received his Master in Computer Science and his Bachelor of Computer Science from University of Brasília (UnB), DF, Brazil, in 2008 and 2003, respectively. He has been working in the Brazilian Office of the Comptroller General (CGU) as an IT expert since 2005 and at UnB as a Professor on the Applied Computer Science Masters program since 2012, when it was created. From 2011 to 2012 he participated in the Transparency Portal team, where his key role was to be the main expert in Open Government Data (OGD). At the end of 2012 he started working as the leader of the Data Science team at the Department of Research and Strategic Information (DIE). 
Mouenis Anouar Tadlaoui et al.

One of the projects developed at DIE, the Reference Price Database, won the first place on the CONIP 2013 Excellence Award in the category Management and Geographical Information Systems. He has done research on fraud detection and prevention for the Brazilian Government and situation awareness for the U.S. Navy. With almost 11 years of experience in the area, he has produced more than 80 different technical outputs, including papers, book chapters, technical presentations, processes, among others. He is an artificial intelligence researcher to focus on data mining, uncertainty in the Semantic Web using Bayesian inference, software engineering, and both $\mathrm{R}$ and Java programming. Awarded programmer with experience in implementation of Bayesian network systems (UnBBayes), multi-entity Bayesian network (MEBN) and Probabilistic Web Ontology Language (PR-OWL), R packages, and various web-based applications.

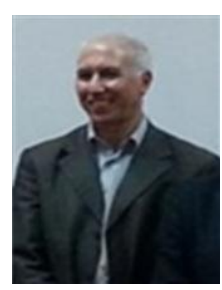

Mohamed Khaldi is a professor at the The École Normale Supérieure at Abdelmalek Essaadi University, and he is with the Laboratory of Informatics, Research Operational and Statistic Applied (LIROSA) at Faculty of Sciences, Abdelmalek Essaadi University. Tétouan, Morocco. 